Cite this: RSC Adv., 2018, 8, 31700

Received 26th June 2018

Accepted 31st August 2018

DOI: $10.1039 / c 8 r a 05484 c$

rsc.li/rsc-advances
Check for updates

\section{Mitochondria targeting IR780-based nanoGUMBOS for enhanced selective toxicity towards cancer cells $\dagger$}

\author{
Mi Chen, ${ }^{a}$ Nimisha Bhattarai, ${ }^{a}$ Mingyan Cong, ${ }^{a}$ Rocío L. Pérez, ${ }^{a}$ Karen C. McDonough ${ }^{b}$ \\ and Isiah M. Warner (D) *a
}

\begin{abstract}
Herein, a simple counter-ion variation strategy is proposed and demonstrated for design of an array of near infrared IR780-based nanoGUMBOS (nanomaterials from a Group of Uniform Materials Based on Organic Salts) to produce enhanced anticancer activity. These nanomaterials were synthesized by direct nanoengineering of IR780-based GUMBOS using a reprecipitation method, without addition of any other materials. Thus, these novel nanomaterials can serve as carrier-free nanodrugs, providing several distinct advantages over conventional chemotherapeutics. Examination of the size and stability of these nanoGUMBOS indicates formation of approximately $100 \mathrm{~nm}$ nanoparticles that are stable under biological conditions. Interestingly, in vitro chemotherapeutic applications of these nanoGUMBOS indicate two to four-fold enhanced toxicity towards breast cancer cells as compared to the parent dye, while still maintaining minimal toxicity towards normal cells. The mechanism of cancer toxicity for these nanoGUMBOS was also examined by a study of their sub-cellular localization as well as using a mitochondrial toxicity assay. Analyses of data from these studies revealed that all nanoGUMBOS primarily accumulate in the mitochondria of cancer cells and produce dysfunction in the mitochondria to induce cell death. Using these studies, we demonstrate tunable properties of IR780-based nanoGUMBOS through simple variation of counter-ions, thus providing a promising strategy for future design of better nanomedicines to be used for cancer therapy.
\end{abstract}

\section{Introduction}

Cancer remains a leading cause of death worldwide. ${ }^{1}$ Much effort has been devoted toward development of novel therapeutics for highly effective cancer therapy. ${ }^{2-4}$ Chemotherapy, one of the most common and efficient cancer treatments, has progressed considerably over the past few decades. However, many issues such as drug resistance and systemic toxicity arise with use of conventional chemotherapeutics. ${ }^{5,6}$ Many mechanisms can be identified for drug resistance including enhanced DNA repair, decreased drug activation, and increased drug degradation. ${ }^{7}$ The latter systemic toxicity can be attributed to lack of selective toxicity of most chemotherapeutic drugs due to non-targeted distribution through the body. This problem ultimately leads to destruction of much of the body's healthy

${ }^{a}$ Department of Chemistry, Louisiana State University, Baton Rouge, LA 70803, USA. E-mail: iwarner@lsu.edu

${ }^{b}$ AgCenter Biotechnology Labs, Louisiana State University, Baton Rouge, LA 70803, USA

$\dagger$ Electronic supplementary information (ESI) available: Detailed experimental methods, chemical structures of all compounds, characterization of GUMBOS and nanoGUMBOS, figures, and tables for support of the discussion. See DOI: $10.1039 / \mathrm{c} 8 \mathrm{ra} 05484 \mathrm{c}$ tissue along with treatment of the tumor. ${ }^{2-4}$ Therefore, it is essential to develop more efficient and selective chemotherapeutics for cancer treatments.

Mitochondria are special subcellular organelles that serve as the main powerhouse of cell. They play a significant role in regulation of cellular metabolism, calcium homeostasis, and programmed cell death. ${ }^{8}$ Importantly, cancer cells have a more hyperpolarized mitochondrial membrane potential $\left(\Delta \Psi_{\mathrm{m}}\right)$ in comparison with normal cells, which makes the uptake of cationic drugs into the cancer cells more favorable. ${ }^{9}$ As a result, targeting of mitochondria has emerged as an attractive strategy for efficient and selective cancer chemotherapy. ${ }^{\mathbf{1 0 1 1}}$ Recently, a lipophilic near-infrared (NIR) heptamethine dye, IR780, has been shown to have promising anticancer characteristics that acts on mitochondria. Free IR780 has been shown to be internalized through organic anion transporter polypeptides (OATPs), and thus cause mitochondrial dysfunction, ultimately inducing cell apoptosis. ${ }^{12,13}$ Despite the anticancer characteristics of this cationic dye, very little research has focused on the application of IR780 as a possible chemotherapeutic agent. Since IR780 has intense fluorescence under NIR excitation, it has primarily been used as a theranostic agent for cell imaging, photodynamic therapy (PDT), and photothermal therapy (PTT). ${ }^{\mathbf{1 4 - 2 0}}$ However, low bioavailability and non-selective 
toxicity at high concentrations have limited its further biological applications. ${ }^{18}$ To address these issues, various nanoscale delivery systems have been developed to encapsulate IR780 in order to minimize systemic toxicity through passive targeting mechanisms such as enhanced permeability and retention effect (EPR). These delivery systems involve use of micelles, polymeric nanoparticles, or quantum dots. ${ }^{\mathbf{1 4 - 2 0}}$ However, there are several challenges when using nanoscale drug carriers, including complexity of nanoparticle fabrication, limited drugloading, and difficulties in controlled drug release at the tumor site. ${ }^{14,16-19,21}$ In this regard, a new concept of carrier-free nanodrugs has been reported, which involves fabrication of nanomaterials using the anticancer drug itself as a chemotherapeutic material and without introduction of drug carrier materials. ${ }^{2,5,6}$ For example, hydrophobic drug molecules can undergo self-assembly to form nanodrugs via change from organic solvent to a non-solvent system, such as aqueous solution. ${ }^{22,23}$ Several nanodrugs have already been approved for clinical trial due to the ultra-high drug loading and reduced material-related toxicity of this approach. ${ }^{24}$ In order to promote the wide application of nanodrugs for clinical cancer therapy, it is highly desirable to design nanodrugs with easy synthesis, good physiological stability, and highly selective toxicity towards cancer cells relative to normal cells.

In recent years, a group of uniform materials based on organic salts (GUMBOS) has been developed in our laboratory for several biomedical applications., ${ }^{25-27}$ Although similar to ionic liquids in terms of counter-ions used, GUMBOS are solidphase analogs with a defined melting point range of $25^{\circ} \mathrm{C}$ to $250{ }^{\circ} \mathrm{C}$. Such organic salts are found to have broadly tunable properties such as toxicity, hydrophobicity, melting point, and optical behaviors. ${ }^{\mathbf{2 , 2 5 , 2 6 , 2 8 , 2 9}}$ Thus, GUMBOS can be designed for specific tasks through simple counter-ion variations. Nanoparticles derived from these GUMBOS (nanoGUMBOS) generally display enhanced, unique, and tunable properties at the nanoscale level as compared to GUMBOS. Specifically, conversion of hydrophobic GUMBOS into nanoGUMBOS for therapeutic applications can enhance bioavailability of therapeutic agents due to the small size and large surface area of nanoGUMBOS. It has also been acknowledged that nanoparticles aid in achieving passive tumor targeting due to the EPR effect of the nanoscale size, which can make nanoGUMBOS more favorable as potential chemotherapeutic drugs in comparison with GUMBOS. ${ }^{30}$ Furthermore, the ease of synthesis of such nanoGUMBOS allows for development of a novel carrier-free nanodrug without addition of other matrices.

In this present study, we aim to enhance the selective cytotoxicity of IR780 for future chemotherapeutic applications through conversion to GUMBOS and nanoGUMBOS. By use of certain advantages of the nanodrug, as well as the GUMBOS concept, we have fabricated a series of IR780-based GUMBOS ([IR780][Asc], [IR780][OTf], [IR780][BETI]) through anionexchange of [IR780][I] with various bulky organic counteranions. These selected anions have varying compositions, size, and hydrophobic properties. Additionally, no cytotoxicity towards cancer or normal cells has been observed for these anions under studied conditions. ${ }^{2}$ Characterization of these
GUMBOS by use of parameters such as hydrophobicity, melting point, absorption, and fluorescence properties was performed. Quasi-spherical nanoGUMBOS were then obtained by directly nanoengineering these GUMBOS using an ultrasonicationassisted reprecipitation method, which has been previously described., 2,23,25,26,31 These nanomaterials were then tested in vitro using both cancer cells and normal cells to study drug efficacy. Examination of results from these studies indicate that these novel NIR nanomaterials provide unique properties for future in vivo chemotherapeutic applications.

\section{Results and discussions}

\section{Synthesis and characterization of IR780-based GUMBOS}

All GUMBOS presented in this work were synthesized by use of a simple metathesis reaction, where the anion of IR-780 was exchanged with a variety of bulky organic counter-anions (structures provided in Fig. $\mathrm{S} 1 \dagger$ ). One scheme of a representative metathesis reaction between [IR780][I] and lithium bis(perfluoroethylsulfonyl) ([Li][BETI]) is shown in Fig. 1. Detailed synthetic procedures are provided in ESI. $\dagger$ All GUMBOS obtained were then subjected to electrospray ionization mass spectrometry (ESI-MS) for confirmation of synthesis. The results from ESI-MS provided in the ESI (Fig. S2, Table S1 $\dagger$ ) were consistent with theoretical calculations.

As expected, the synthesized IR780-GUMBOS displayed variable physicochemical properties with counter-anion variation, including properties such as melting point, hydrophobicity, and optical behaviors (Table S2 $\dagger$ ). Among these properties, hydrophobicity, which affects the nanoparticle formation process and subsequent stabilization, of IR780-based GUMBOS was estimated by use of octanol/water partition coefficients $(\log P)$ (Table $\mathrm{S} 2 \dagger){ }^{22}$ Comparison of anion variation indicates that [IR780][BETI] GUMBOS is the most hydrophobic, followed by [IR780][I], [IR780] [OTf], and [IR780][Asc]. Such differences in hydrophobicity is quite dependent upon the counter-anion, which corroborates previous literature findings. ${ }^{2,25}$ In addition, optical behaviors of GUMBOS, including both absorption and fluorescence spectra measured in acetonitrile, were evaluated and depicted in Fig. 2(a) and (b), respectively. All GUMBOS displayed similar absorption and emission spectra as the parent compound. The absorption maximum was observed at $780 \mathrm{~nm}$ with a shoulder at $710 \mathrm{~nm}$. The emission maximum was measured to be $800 \mathrm{~nm}$. Observed differences in absorbance and fluorescence intensities of GUMBOS are likely a result of effects from anion variations. These IR780 based GUMBOS also exhibited good NIR radiation absorption and strong fluorescence emission similar to the parent dye. Such properties allow direct visualization of the distribution of GUMBOS in cells or tissues through near infrared fluorescence (NIRF) imaging. ${ }^{32,33}$

\section{Synthesis and characterization of IR780-based nanoGUMBOS}

Due to the hydrophobic properties of IR780-based GUMBOS, nanoGUMBOS were prepared by use of an additive free reprecipitation method in conjunction with ultra-sonication. ${ }^{25}$ Briefly, a small aliquot of GUMBOS dissolved in dimethyl sulfoxide (DMSO) was rapidly injected into a large amount of 
<smiles>CCCN1/C(=C/C=C2\CCCC(/C=C/C3=[N+](CCC)C(C)(C)c4ccccc4C3(C)C)=C2Cl)C(C)(C)c2ccccc21</smiles><smiles>O=S(=O)(NS(=O)(=O)C(F)(F)C(F)(F)F)C(F)(F)C(F)(F)F</smiles>

$\mathrm{Li}^{+}$

[IR780][l]

DCM/Water

[Li][BETI]

stir for $48 \mathrm{~h}$ at r.t.

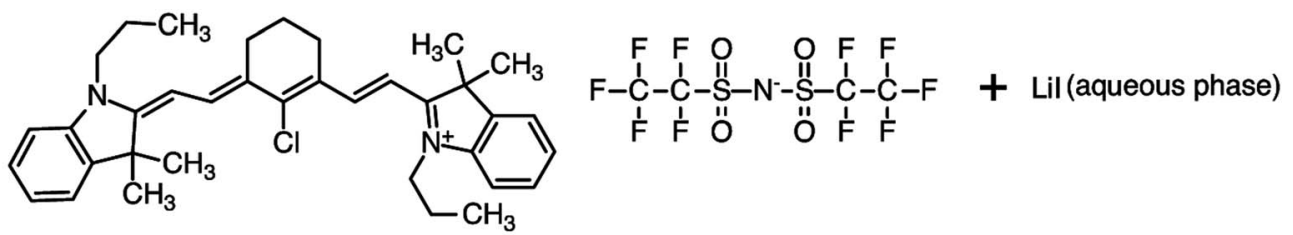

[IR780][BETI]

Fig. 1 Synthesis of [IR780][BETI] GUMBOS by use of anion exchange.
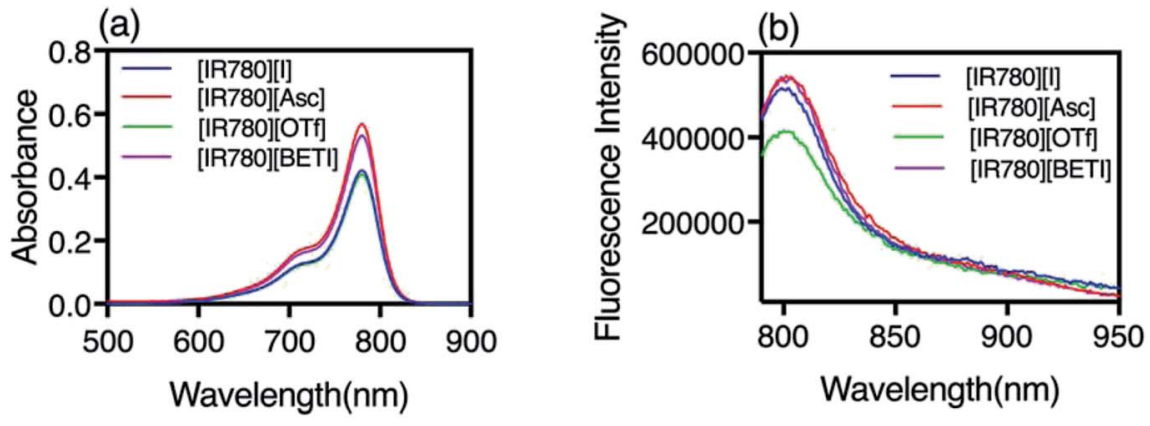

Fig. 2 (a) Absorbance spectra and (b) fluorescence emission spectra of IR780 and IR780-based GUMBOS in acetonitrile.

aqueous cell medium (Dul-becco Modified Eagel Medium (DMEM); containing $10 \%$ fetal bovine serum) under ultrasonication. In this scenario, a high super saturation was reached, thus initiating self-assembly of hydrophobic GUMBOS to form nanoparticles. The resulting nanoparticle size was controlled by simple variation in initial concentration of hydrophobic GUMBOS, which has also been used in fabrication of other size-controlled hydrophobic nanodrugs..$^{22,34}$ As a result, the size of different nanoGUMBOS was optimized to approximately $100 \mathrm{~nm}$ in this manner, which is considered excellent for tumor targeting and accumulations owing to EPR effect. ${ }^{35,36}$ Quasi-spherical or slightly ovate shapes were measured for resulting nanoparticles by use of transmission electron microscopy (TEM). A histogram of the TEM sizes are summarized in Fig. 3(a-d), showing a narrow distribution of the actual size of sample in a dried state. In addition, the hydrodynamic size of the nanoparticles was evaluated in phosphate buffered saline (PBS) using dynamic light scattering (DLS). As shown in Fig. S3, $\uparrow$ all measured nanomaterials displayed a hydrodynamic size that is larger than $200 \mathrm{~nm}$ due to aggregation in an aqueous solution system. Moreover, DLS analysis revealed that the most hydrophobic GUMBOS, [IR780][BETI], yielded the most uniform nanoGUMBOS with a polydispersity index of 0.12 (Fig. S3†). Such results also confirm the predominant role of hydrophobicity on nanoparticle formation using a reprecipitation method as discussed previously. ${ }^{22}$ Measurements of zeta potential suggest that all nanoGUMBOS displayed a negative surface charge in PBS (Table S3†). This indicates that the surfaces of the nanoparticles are predominately anion controlled.

Considering the application of IR780-based nanoGUMBOS in biological systems, spectral behavior of these nanoparticles in serum-DMEM $(10 \%$ FBS in DMEM, $\mathrm{pH}=7.4)$ was also evaluated (Fig. 4). Intriguingly, the relatively less hydrophobic [IR780][Asc] and [IR780][OTf] nanoGUMBOS exhibited similar absorption spectra profile to the parent dye based nanoparticles, where peak absorbance occurs at $790 \mathrm{~nm}$ with a shoulder at $720 \mathrm{~nm}$. In contrast, for the most hydrophobic compound, [IR780][BETI], the nanoparticle suspensions in serum-DMEM displayed a slightly broader absorption spectra. This broadening from $820 \mathrm{~nm}$ to $900 \mathrm{~nm}$ is consistent with formation of J-type aggregation as a result of staircase head-to- 
(a)

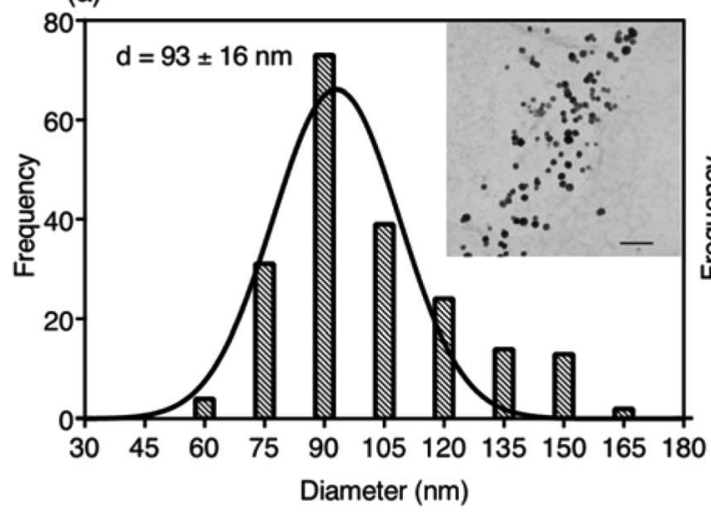

(b)

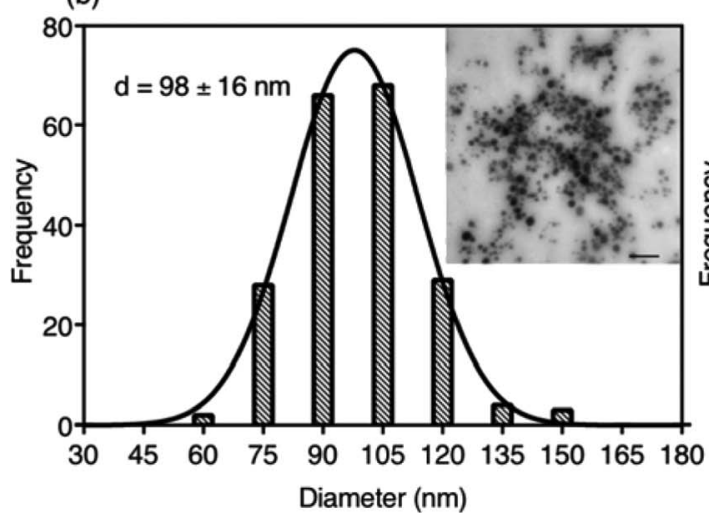

(c)

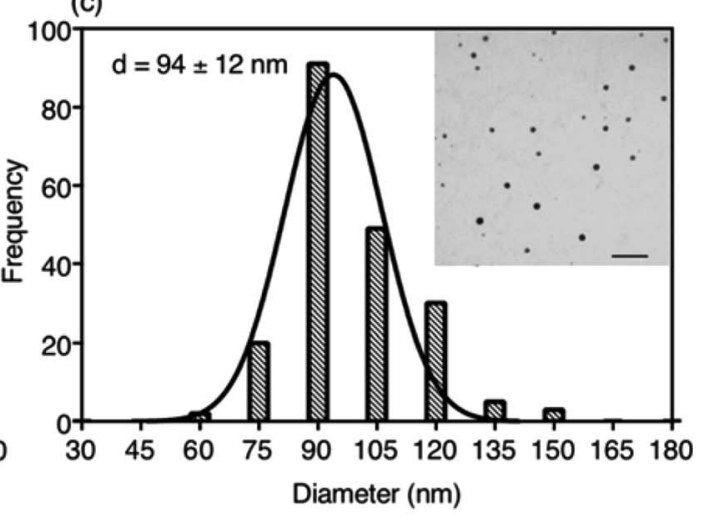

(d)

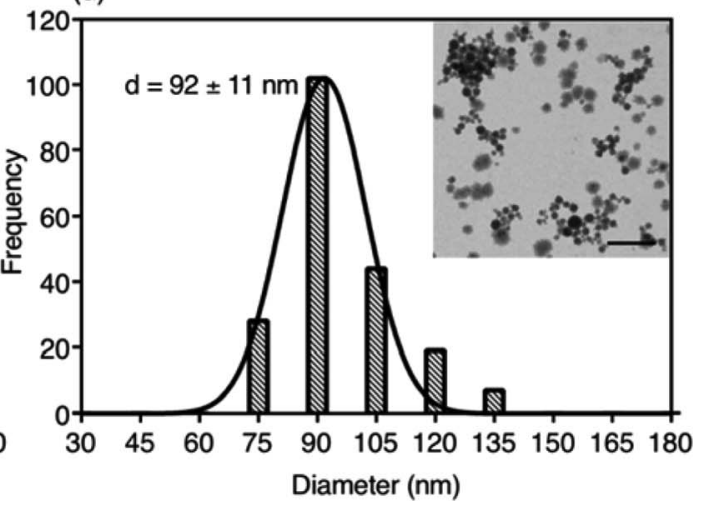

Fig. 3 Characterization of particle sizes for IR780-based nanoparticles using TEM. Histograms constitutes 200 individual nanoparticles with a distribution curve overlay as well as a representative portion of a TEM micrograph (all scale bars represent 500 nm): (a) [IR780][I], (b) [IR780] [Asc], (c) [IR780][OTf], (d) [IR780][BETI].

tail arrangement of transition dipoles. ${ }^{37}$ All nanoparticles also displayed similar fluorescence spectrum to each other with peak emission near $810 \mathrm{~nm}$, which is in the desirable NIR region. This property allows for NIRF imaging, making nanoGUMBOS more suitable for in vivo studies of bio-distribution due to deep tissue penetration and low autofluorescence in comparison with many previously reported fluorescent nanoparticles for chemotherapeutic applications. ${ }^{38-41}$ Of all nanoGUMBOS studied, [IR780][Asc] and [IR780][BETI] have even shown more intense emission signal in comparison with the parent dye. In addition, when compared to free IR780 in acetonitrile, a $10 \mathrm{~nm}$ red-shift was observed for all investigated IR780-based nanoparticles in terms of both absorption and emission spectra. This results from either a change in solvent polarity or from strong hydrophobic interactions between IR780 and other molecules in the serum-DMEM, which is consistent with previous literature data. ${ }^{15,17}$

Following initial photochemical examinations of these nanoGUMBOS, the colloidal stabilities of nanoparticles in cell culture medium were investigated since they play a profound role in cellular uptake as well as cytotoxicity. ${ }^{42}$ A UV-Vis NIR spectrophotometer was used to monitor absorbance spectra of all IR780based nanoparticles in serum-DMEM over a $48 \mathrm{~h}$ period as presented in Fig. S4. $\dagger$ It is interesting to note that an increase in peak absorbance at $790 \mathrm{~nm}$ corresponding to randomly oriented aggregates was observed initially, with a decrease in absorbance from $820 \mathrm{~nm}$ to $850 \mathrm{~nm}$ corresponding to J-aggregates. This indicates dye-deaggregation, where J-aggregation has shifted to randomly oriented aggregation. Similar results from previous studies of R6G nanoparticles have also been observed. ${ }^{2}$ In the subsequent hours, little or no change of spectra were observed (Fig. 4(c) and S4 †). This suggests formation of stable nanoparticles under biological conditions, in which nanoparticles were prevented from non-specific adsorption on the walls of quartz cuvettes and stabilized in the presence of proteins in serum-DMEM. Additionally, examination of size and shape of nanoGUMBOS after $48 \mathrm{~h}$ of storage in cell culture media were also performed by use of TEM measurements. For example, as shown in Fig. S5, $\uparrow$ the sizes of all nanoGUMBOS were still approximately $100 \mathrm{~nm}$ with spherical shapes after $48 \mathrm{~h}$ of storage in cell culture medium. Neither size, nor morphology of nanoGUMBOS varied with time, which thus proved stability of these nanoparticles in cell culture medium. This favorable stability of IR780-based nanoGUMBOS in cell culture medium suggests their potential use for future in vivo applications.

\section{In vitro cytotoxicity evaluation of IR780-based nanoGUMBOS}

In vitro cytotoxicity of the parent [IR780][I] nanoparticles and the most uniform [IR780][BETI] nanoGUMBOS were first 

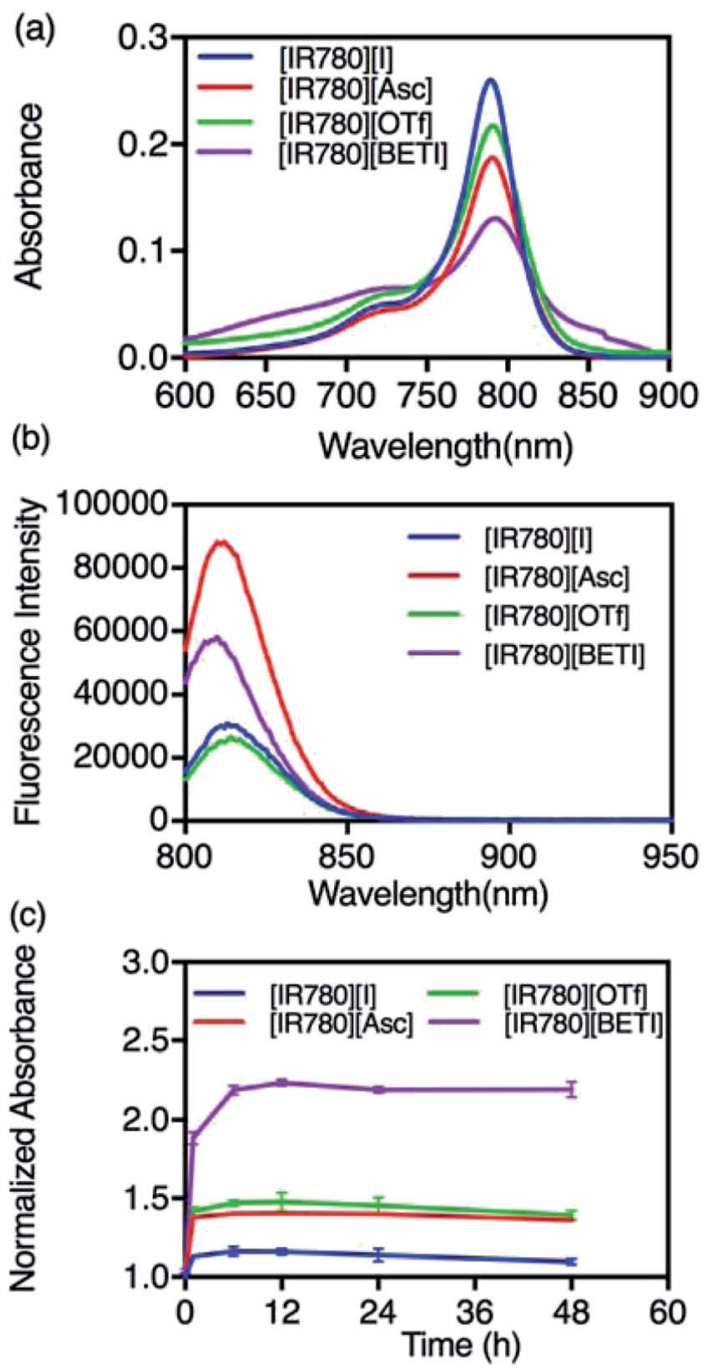

Fig. 4 (a) Absorbance spectra, (b) fluorescence emission spectra of IR780-based nanoparticles, and (c) stability of IR780-based nanoparticles in serum-DMEM. Normalized absorbance was determined by dividing the peak absorbance after certain hours by the initial peak absorbance.

evaluated using three different human cancer cell lines including breast cancer (MDA-MB-231, MCF-7) and pancreatic cancer (MIA PaCa-2). These cells were incubated using various concentrations of IR780-based nanoparticles for $48 \mathrm{~h}$. Subsequently, cell viability was examined and quantified using a colorimetric methyltetrazolium (MTT) viability assay. The $\mathrm{IC}_{50}$ values of these cancer cells, which represent the concentration of drug at $50 \%$ cell viability, were then calculated and compared. Fig. 5(a) is a graph of $\mathrm{IC}_{50}$ values of parent [IR780][I] nanoparticles and [IR780][BETI] nanoGUMBOS for these different cancer cell lines. The $\mathrm{IC}_{50}$ values of [IR780][BETI] nanoGUMBOS for the three cancer cell lines were significantly smaller as compared with the parent dye, suggesting higher cytotoxicity of nanoGUMBOS. Moreover, these results clearly reveal that among all the tested cancer cell lines, the aggressive and invasive MDA-MB-231 breast cancer cell line, was most susceptible to the parent IR780 nanoparticles as well as [IR780] [BETI] nanoGUMBOS.
The MDA-MB-231 breast cancer cells were used to further study the differences in therapeutic efficacy of all synthesized IR780-based nanoGUMBOS. As shown in Fig. 5(b), a significant reduction in $\mathrm{IC}_{50}$ concentration as compared to the parent dye was observed for all investigated nanoGUMBOS. The [IR780] [BETI] nanoGUMBOS were found to be most toxic to cancer cells with the lowest $\mathrm{IC}_{50}$ value of $4.6 \mu \mathrm{M}$. This [IR780][BETI] nanoGUMBOS had more than a threefold reduction in $\mathrm{IC}_{50}$ concentration as compared to the parent [IR780][I], with an $\mathrm{IC}_{50}$ value of $17 \mu \mathrm{M}$. The other two nanoGUMBOS, [IR780][Asc] and [IR780] [OTf] also outperformed the parent dye in terms of anticancer efficacy, showing at least 1.5 fold lower $\mathrm{IC}_{50}$ concentrations. In comparison with R6G based nanoGUMBOS developed previously in our laboratory, IR780 based nanoGUBMBOS have shown enhanced toxicity towards cancer cells. ${ }^{2}$ Analysis of these results demonstrated the proven role of counter-ion variation on the toxicity of such nanomaterials.

Examination of cytotoxicity on normal epithelial breast cells, i.e. Hs578Bst, was performed in order to assess the selectivity of nanoGUMBOS. As presented in Fig. 5(c-f), while all nanoparticles exhibited a dose-dependent inhibition towards breast cancer cell proliferation, only a small impact on the viability of normal breast Hs578Bst was observed under conditions tested. For example, treatment of MDA-MB-231 cancer cells with [IR780][BETI] nanoGUMBOS led to almost complete eradication of the cells at a concentration of $12.5 \mu \mathrm{M}$, while greater than $75 \%$ cell viability of normal cells was still maintained. This suggests good selectivity of [IR780][BETI] nanoGUMBOS towards cancer cells relative to normal cells. It is also interesting to note that all IR780-based nanoGUMBOS displayed an enhanced selective toxicity towards MDA-MB-231 cancer cell in comparison to the parent IR780 nanoparticles, as indicated by their wider gap of cytotoxicity between cancer and normal cells. A similar selective outcome was also observed for MCF-7 breast cancer cell line and HMEC normal epithelial breast cell line (Fig. S6†). In this case, the selective toxicity may be attributed to enhanced cellular uptake of nanoGUMBOS in cancer cells relative to normal cells, as previously reported for the parent IR780 in other cancer cell lines. ${ }^{\mathbf{1 3 , 1 8 , 4 3}}$ This conclusion was further validated by use of microscopy studies in which higher fluorescence intensity was observed in cancer cells relative to normal cells after treatment with the same concentration of nanoGUMBOS (Fig. S7†). Examination of these results suggest that anion exchange with parent compound aided in improvement of anti-tumor effects while still displaying good selectivity in vitro. Thus, our nanoGUMBOS may be good candidates for further exploration as potential therapeutic drugs.

\section{Cellular uptake studies}

Effective cellular uptake plays an important role in enhancing nanoparticle retention, EPR effects, and ultimately therapeutic efficacy. ${ }^{44}$ In order to further understand improved drug efficacy of nanoGUMBOS as compared to the parent dye, the cellular uptake of all nanoparticles with different absorptivity were quantitatively measured and compared using a previously reported method with slight modification. ${ }^{26,45,46}$ An optimized 

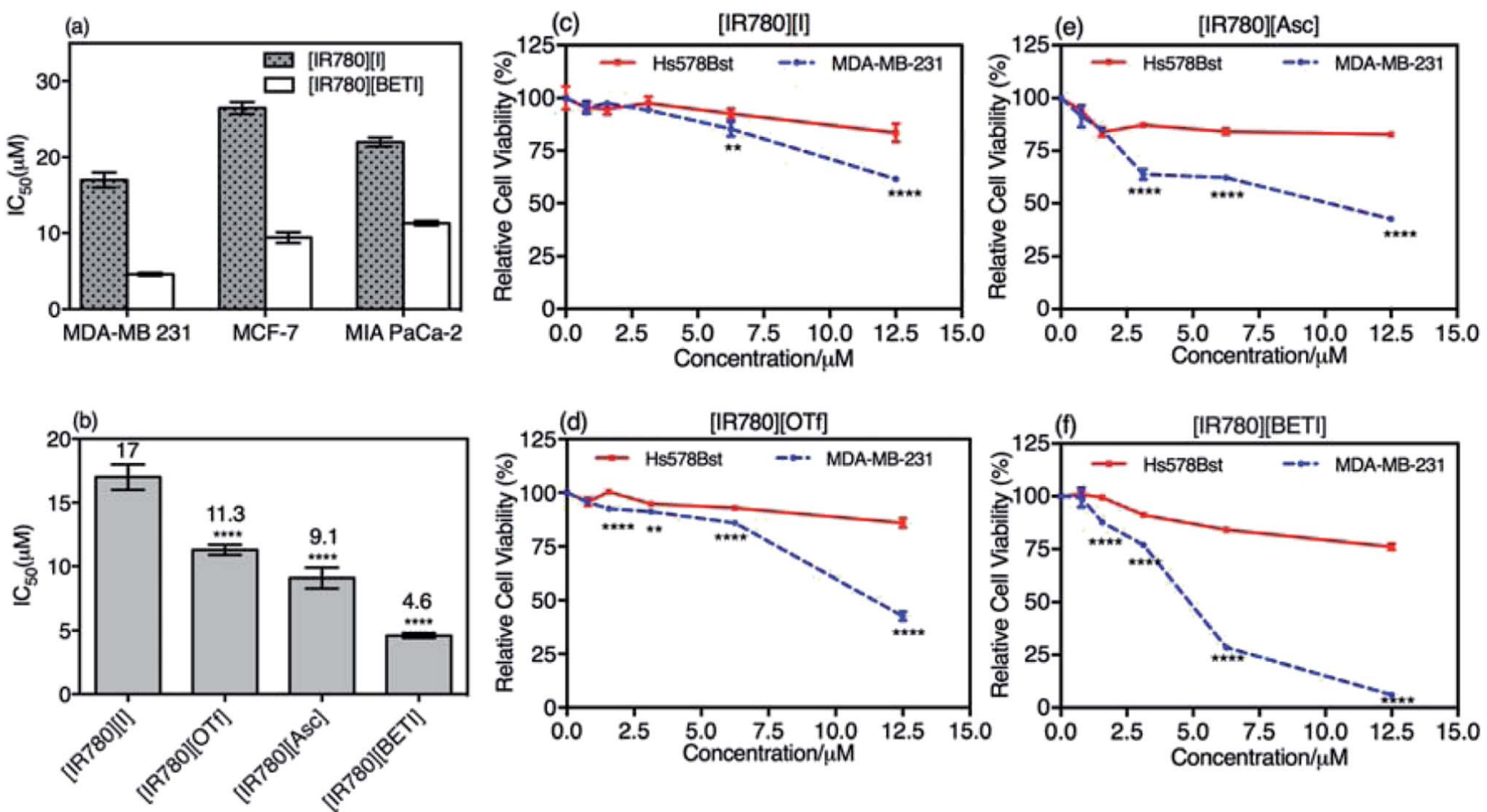

Fig. 5 (a) The IC $\mathrm{C}_{50}$ values of [IR780] [I] nanoparticles and [IR780][BETI] nanoGUMBOS for cultured MDA-MB-231, MCF-7, and MIA PaCa-2 cancer cells. (b) The $I_{50}$ values of different IR780-based nanoparticles for MDA-MB-231. (c)-(f) Cell viability for MDA-MB-231 cancer cell and Hs578Bst normal cell after treatment of IR780-based nanoparticles for $48 \mathrm{~h}$. Data are presented as mean $\pm \mathrm{s}$.d. $(n=6)$. For some points, the error bars are shorter than the symbol, and thus are not visible in the graph. Statistical significance was assessed by use of SPSS via one-way ANOVA test; (**P $\leq$ $0.05, * * * P \leq 0.001, * * * * P \leq 0.0001)$.

concentration of nanoparticles corresponding to little or no cell death was incubated with MDA-MB-231 cancer cells for $4 \mathrm{~h}$, and then cellular uptake of incubated nanoparticles were quantified based on spectrometric measurements. As shown in Fig. 6, all nanoGUMBOS exhibited significantly higher cellular uptake than the original [IR780][I] nanoparticle, which suggests a greater amount of nanoGUMBOS accumulated inside the breast cancer cells. These results further corroborate the observed enhanced cancer killing activity of nanoGUMBOS. Since uptake of nanoparticles commonly occurs through receptor-mediated pathways, this process is largely influenced

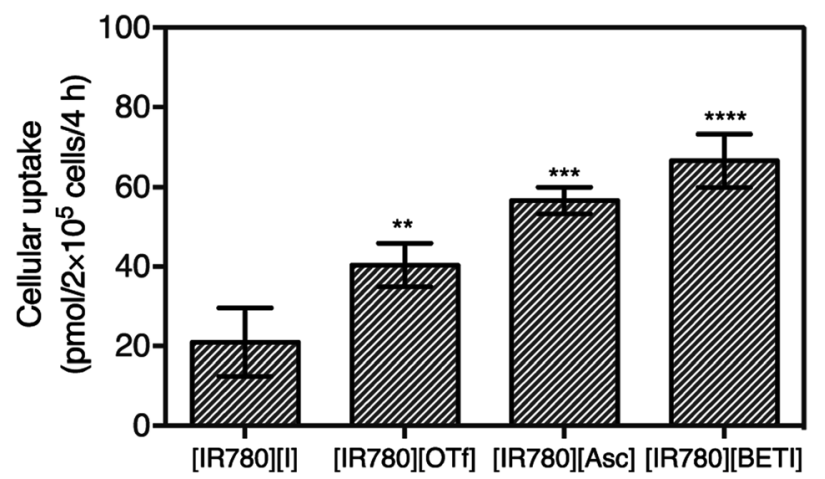

Fig. 6 Cellular uptake of nanoparticles after incubation of $25 \mathrm{nmol}$ NPs with MDA-MB-231 cancer cells. Data are presented as mean \pm s.d. $(n=3)$. Statistical significance was assessed by SPSS via one-way ANOVA test; ( $* * P \leq 0.05, * * * P \leq 0.001, * * * * P \leq 0.0001)$. by the physicochemical properties of nanoparticles including hydrophobicity, size, surface charge, and composition..$^{47,48}$ Therefore, shape and size of all nanoparticles were characterized to ensure that all exhibited similar properties. The internalization of [IR780][I] has previously been demonstrated to depend upon organic anion transporting polypeptides (OATPs), more specifically the OATP1B3 subtype. ${ }^{12,13,18}$ Therefore, we hypothesize that the counter-ion exchange probably promotes interaction with OATPS transporters, leading to higher uptake. Further studies to elucidate the internalization mechanism are ongoing in our laboratory.

\section{Intracellular stability studies}

Due to the complexity of the intracellular environment, in which various enzymes and hydrolases coexist, internalized nanoparticles generally undergo degradation and have a decreased chance of reaching the target organelle in order to exert its function. ${ }^{49}$ Thus, following evaluation of cellular uptake, we then investigated the effect of the intracellular stability of all nanoGUMBOS on drug efficacy. By use of bioTEM, the change of nanoparticles behaviors after internalization into cancer cells was tracked. As indicated in Fig. 7(a), only a few [IR780][I] nanoparticles were observed inside MDA-MB231 cancer cells after $30 \mathrm{~min}$ incubation. This phenomenon most likely resulted from dissociation or degradation of the internalized nanoparticles that has been confirmed previously through detection of strong fluorescence from the parent dye 


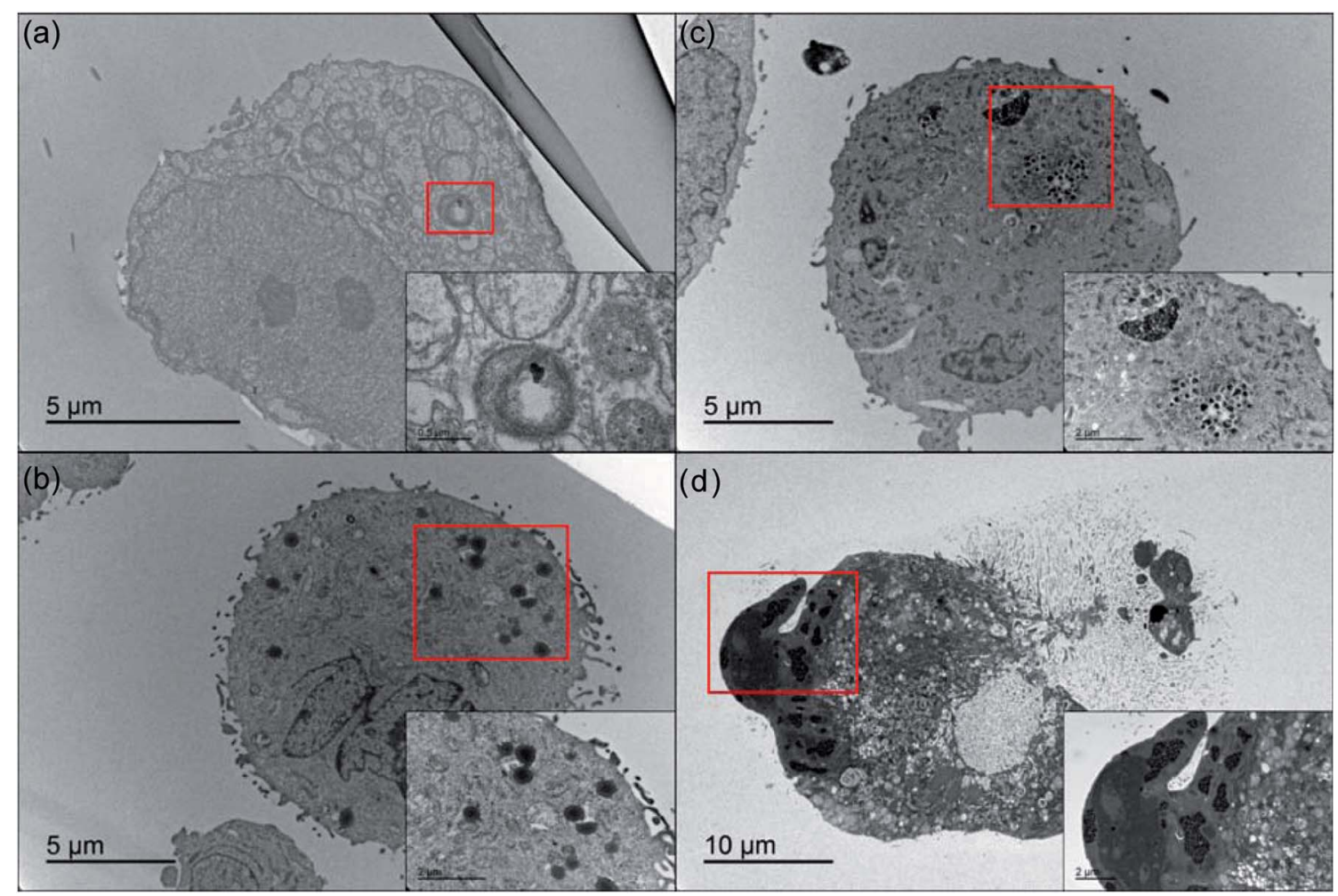

Fig. 7 Bio-TEM images of MDA-MB 231 cells incubated with (a) [IR780][I] nanoparticles, (b) [IR780][OTf], (c) [IR780][Asc], and (d) [IR780][BETI] nanoGUMBOS for $30 \mathrm{~min}$. Insets are the enlarged images corresponding to the red rectangle sections of each image, showing the presence of nanoparticle.

(Fig. S7 $\dagger$ ). In contrast, a large number of IR780 based nanoGUMBOS were present in the cytoplasm and still maintained intact morphology as shown in Fig. 7(c and d). In this regard, rather than undergoing degradation, most nanoGUMBOS proceeded to aggregate into large particles or clusters after internalization into cells. Thus, it is reasonable to expect that the aggregation behavior of nanoGUMBOS helps their retention in cancer cells long enough to reach the target organelle. This is also consistent with enhanced accumulation of nanoGUMBOS as shown in our cellular uptake study.

\section{Mechanism of anticancer effect of IR780-based nanoparticles}

Since all IR780 based nanoGUMBOS have shown efficient cellular uptake, good intracellular stability, and good antitumor activity, the mechanism of cancer toxicity also needs to be determined for future applications. It is known that IR780 dye preferentially accumulates in the mitochondria of cancer cells in order to induce cell apoptosis. ${ }^{13,43}$ In this context, we also examined sub-cellular localization of our nanoGUMBOS (represented as red fluorescence) by co-staining with MitoTracker green dye (represented as green fluorescence) in breast MDA-MB 231 cancer cells. As presented in Fig. 8(a), strong fluorescence from IR780-based nanoGUMBOS was observed indicating the effective internalization of nanoGUMBOS. The merged images show a large yellow overlay of red fluorescence from nanoGUMBOS and green fluorescence from the MitoTracker dye. This suggests that similar to the [IR780][I], all nanoGUMBOS also primarily accumulate in the mitochondria in breast cancer cells, indicating that the mitochondria may also be a major target organelle of IR780-based nanoGUMBOS in order to induce cell death. Additionally, the Pearson's coefficients measuring degree of co-localization of nanoGUMBOS and MitoTracker were calculated to be larger than 0.7 (Table S5 $\dagger$ ), quantitatively confirming the great mitochondria localization of nanoGUMBOS. To elucidate the specific mechanism of cancer toxicity for these nanoGUMBOS, a mitochondrial toxicity assay in combination with the galactose cell culture growth medium was further performed, which allows prediction of potential mitochondrial dysfunction as a result of drug exposure. ${ }^{50}$ This assay consists of two measurements, including cell membrane integrity (MI) associated with cytotoxicity, and cellular ATP levels. Typically, a mitochondria toxin inhibits oxidative phosphorylation, resulting in decreased ATP with no change or disproportional increase in its measured cytotoxicity as compared to the vehicle control. In contrast, a reduction in ATP with a proportional increase in cytotoxicity suggests primary necrosis is occurring instead of mitochondria dysfunction. ${ }^{2}$ As shown in Fig. 8(b), cells treated with these nanoGUMBOS displayed similar results as the parent dye, in which ATP levels were reduced with a disproportional increase in cytotoxicity relative to the vehicle control, indicating all IR780based nanoGUMBOS are also mitochondrial toxins. Similar mitochondria toxicity profiles of other known mitochondrial toxins such as antimycin and rhodamine $6 \mathrm{G}$ were also observed in other literature. ${ }^{2,30}$ Therefore, it can be concluded that cancer toxicity of these nanoGUMBOS, as well as the parent dye arise from inhibition of oxidative phosphorylation in the mitochondria of MDA-MB231 cancer cells. In comparison to other nanoparticles that act through a similar mitochondria-based anticancer route, most of 
(a)

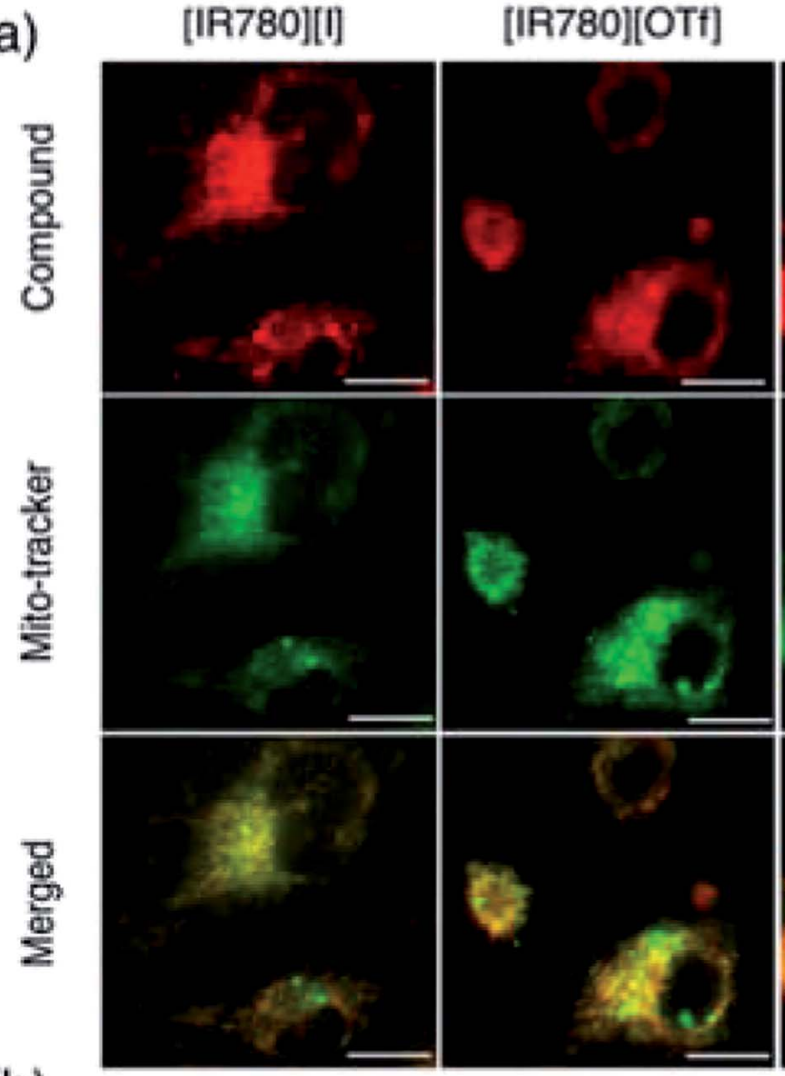

\section{[IR780][Asc]}
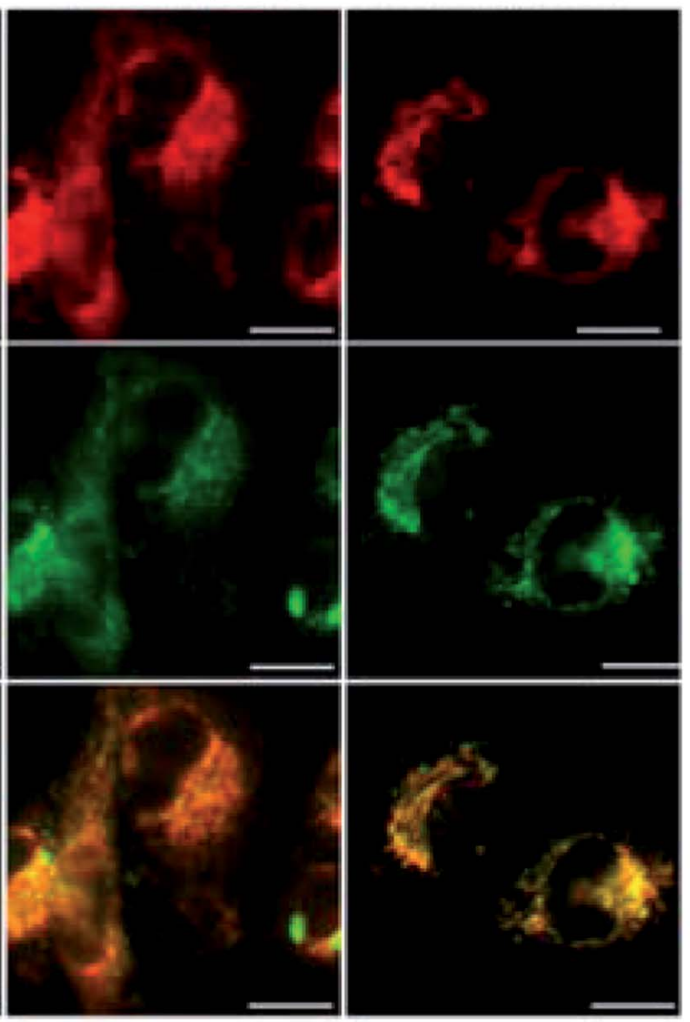

(b)
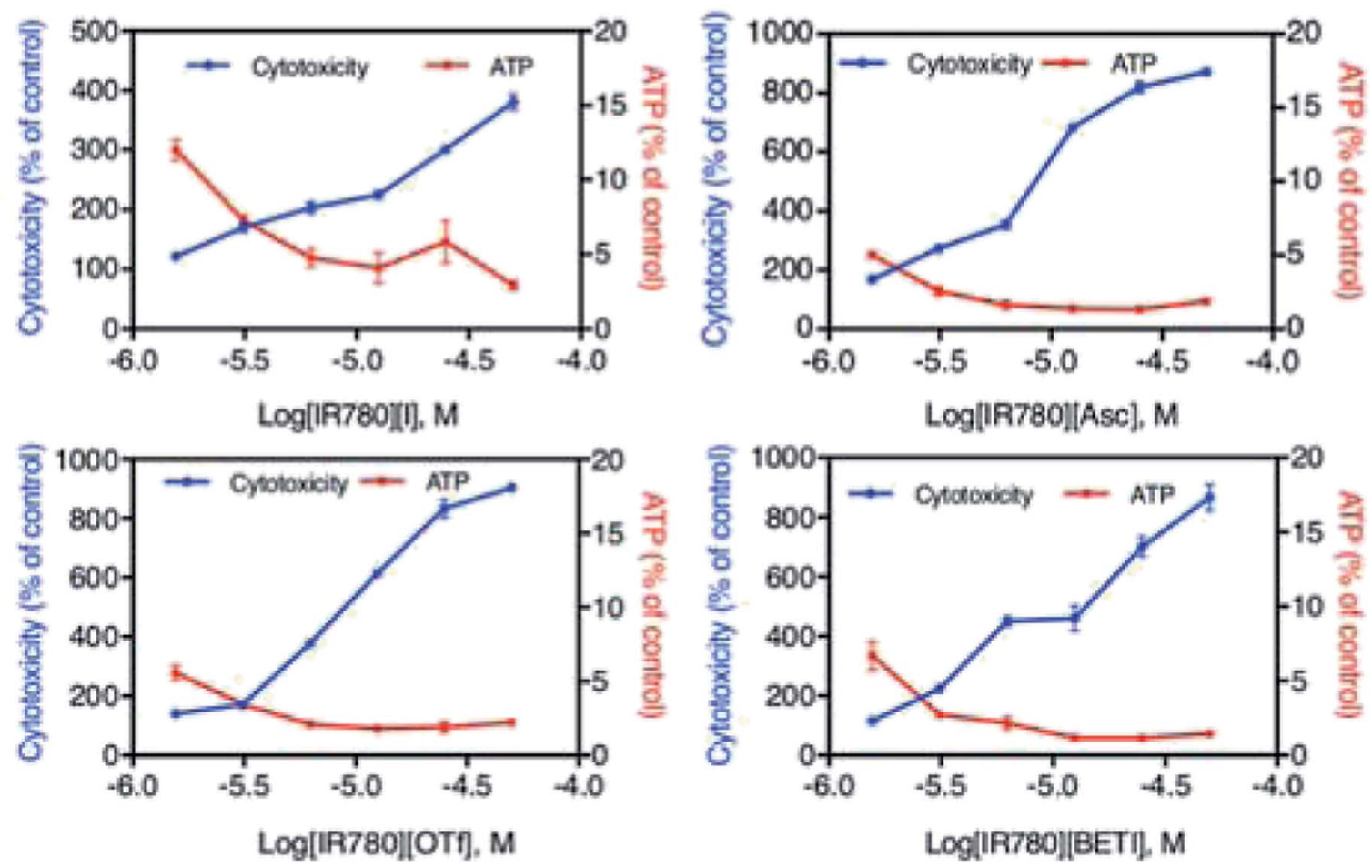

Fig. 8 The uptake of IR780-based nanoGUMBOS and the effect on the mitochondria of MDA-MB-231 cancer cells. (a) Co-localization of IR780based nanoGUMBOS with Mito-Tracker dye imaged by use of fluorescence microscope. All scale bars on the fluorescence microscopy images represent $20 \mu \mathrm{m}$. (b) Profiles of mitochondria toxicity of IR780-based nanoGUMBOS using a mitochondrial ToxGlo ${ }^{\text {TM }}$ assay. ATP level decreasing with disproportional changes in membrane integrity associated with cytotoxicity indicates a mitochondrial toxin. 
the reported literature has involved use of various materials to achieve targeting function..$^{\mathbf{4 0 4 1}}$ In contrast, our IR780 based nanoGUMBOS target the cancer cell mitochondria due to the cationic charge of IR780, and displayed selective toxicity towards cancer cells without use of any other targeting moiety.

\section{Conclusion}

In this study, we outline a simple counter-ion variation strategy to design a series of tunable nanoGUMBOS based on IR780, a known NIR anticancer dye. These novel IR780-based nanoGUMBOS are easily synthesized with a size of approximately $100 \mathrm{~nm}$. Moreover, our nanoGUMBOS possess high stability in true biological systems as observed in the bio-TEM study. Most importantly, all nanoGUMBOS displayed enhanced selective cytotoxicity towards breast cancer cells in the absence of targeting molecules when compared with the parent dye. Comparison of cytotoxicity results of all nanoGUMBOS suggests that [IR780][BETI] displays the best drug efficacy towards MDA-MB-231 breast cancer cells with minimum toxicity towards normal breast cells, followed by [IR780][Asc], and then [IR780][OTf]. Mitochondrial assays revealed that the anticancer effects of the nanoGUMBOS results from mitochondria dysfunction. In conclusion, our findings provide deep insight and encourage further investigations of IR780-based nanoGUMBOS as chemotherapeutic agents, as well as use in combination with PDT or PTT for improved cancer therapy.

\section{Conflicts of interest}

There are no conflicts to declare.

\section{Acknowledgements}

We thank Ms Ying Xiao for helping with the bio-TEM study. We are also grateful to Dr Suzana Hamdan, Dr Noureen Siraj, and Dr Pratap Chhotaray for useful suggestions and discussions regarding this manuscript. This material is based upon work supported in part by the National Science Foundation under Grants CHE-1307611 and No. CHE-1508726. Any opinions, findings, and conclusions or recommendations expressed in this material are those of the author(s) and do not necessarily reflect the views of the National Science Foundation.

\section{References}

1 R. Siegel, D. Naishadham and A. Jemal, CA-Cancer J. Clin., 2013, 63, 11-30.

2 P. K. S. Magut, S. Das, V. E. Fernand, J. Losso, K. McDonough, B. M. Naylor, S. Aggarwal and I. M. Warner, J. Am. Chem. Soc., 2013, 135, 15873-15879.

3 K. D. Miller, R. L. Siegel, C. C. Lin, A. B. Mariotto, J. L. Kramer, J. H. Rowland, K. D. Stein, R. Alteri and A. Jemal, CA-Cancer J. Clin., 2016, 66, 271-289.

4 R. W. Humphrey, L. M. Brockway-Lunardi, D. T. Bonk, K. M. Dohoney, J. H. Doroshow, S. J. Meech, M. J. Ratain, S. L. Topalian and D. M. Pardoll, J. Natl. Cancer Inst., 2011, 103, 1222-1226.
5 P. Huang, D. Wang, Y. Su, W. Huang, Y. Zhou, D. Cui, X. Zhu and D. Yan, J. Am. Chem. Soc., 2014, 136, 11748-11756.

6 Y. Li, J. Lin, Y. Huang, Y. Li, X. Yang, H. Wu, S. Wu, L. Xie, L. Dai and Z. Hou, ACS Appl. Mater. Interfaces, 2015, 7, 25553-25559.

7 Y. A. Luqmani, Med. Princ. Pract., 2005, 14, 35-48.

8 S. Wen, D. Zhu and P. Huang, Future Med. Chem., 2013, 5, 5367.

9 C. Yue, Y. Yang, J. Song, G. Alfranca, C. Zhang, Q. Zhang, T. Yin, F. Pan, J. M. de la Fuente and D. Cui, Nanoscale, 2017, 9, 11103-11118.

10 W.-Q. Li, Z. Wang, S. Hao, H. He, Y. Wan, C. Zhu, L.-P. Sun, G. Cheng and S.-Y. Zheng, ACS Appl. Mater. Interfaces, 2017, 9, 16793-16802.

11 A. Mallick, P. More, S. Ghosh, R. Chippalkatti, B. A. Chopade, M. Lahiri and S. Basu, ACS Appl. Mater. Interfaces, 2015, 7, 7584-7598.

12 Y. Wang, T. Liu, E. Zhang, S. Luo, X. Tan and C. Shi, Biomaterials, 2014, 35, 4116-4124.

13 E. Zhang, S. Luo, X. Tan and C. Shi, Biomaterials, 2014, 35, 771-778.

14 F. Guo, M. Yu, J. Wang, F. Tan and N. Li, ACS Appl. Mater. Interfaces, 2015, 7, 20556-20567.

15 C. Jiang, H. Cheng, A. Yuan, X. Tang, J. Wu and Y. Hu, Acta Biomater., 2015, 14, 61-69.

16 S. Li, S. Zhou, Y. Li, X. Li, J. Zhu, L. Fan and S. Yang, ACS Appl. Mater. Interfaces, 2017, 9, 22332-22341.

17 K. Wang, Y. Zhang, J. Wang, A. Yuan, M. Sun, J. Wu and Y. Hu, Sci. Rep., 2016, 6, 27421.

18 X. Yi, F. Yan, F. Wang, W. Qin, G. Wu, X. Yang, C. Shao, L. W. K. Chung and J. Yuan, Med. Sci. Monit., 2015, 21, 511-517.

19 A. Yuan, X. Qiu, X. Tang, W. Liu, J. Wu and Y. Hu, Biomaterials, 2015, 51, 184-193.

20 C. Yue, P. Liu, M. Zheng, P. Zhao, Y. Wang, Y. Ma and L. Cai, Biomaterials, 2013, 34, 6853-6861.

21 J. D. Rocca, D. Liu and W. Lin, Nanomedicine, 2012, 7, 303305.

22 S. M. D'Addio and R. K. Prud'homme, Adv. Drug Delivery Rev., 2011, 63, 417-426.

23 H. Kasai, T. Murakami, Y. Ikuta, Y. Koseki, K. Baba, H. Oikawa, H. Nakanishi, M. Okada, M. Shoji, M. Ueda, H. Imahori and M. Hashida, Angew. Chem., Int. Ed., 2012, 51, 10315-10318.

24 J.-U. A. H. Junghanns and R. H. Müller, Int. J. Nanomed., 2008, 3, 295-310.

25 D. K. Bwambok, B. El-Zahab, S. K. Challa, M. Li, L. Chandler, G. A. Baker and I. M. Warner, ACS Nano, 2009, 3, 3854.

26 J. C. Dumke, A. Qureshi, S. Hamdan, K. Rupnik, B. El-Zahab, D. J. Hayes and I. M. Warner, Photochem. Photobiol. Sci., 2014, 13, 1270-1280.

27 M. Cong, N. Siraj, N. Bhattarai, P. E. Kolic, K. S. McCarter, P. K. Chhotaray and I. M. Warner, Sens. Actuators, B, 2018, 257, 993-1000.

28 N. Siraj, F. Hasan, S. Das, L. W. Kiruri, K. E. Steege Gall, G. A. Baker and I. M. Warner, J. Phys. Chem. C, 2014, 118, 2312-2320. 
29 I. M. Warner, B. El-Zahab and N. Siraj, Anal. Chem., 2014, 86, 7184-7191.

30 L. Kamalian, A. E. Chadwick, M. Bayliss, N. S. French, M. Monshouwer, J. Snoeys and B. K. Park, Toxicol. In Vitro, 2015, 29, 732-740.

31 S. Hamdan, J. C. Dumke, B. El-Zahab, S. Das, D. Boldor, G. A. Baker and I. M. Warner, J. Colloid Interface Sci., 2015, 446, 163-169.

32 H. Wang, R.-l. Han, L.-m. Yang, J.-h. Shi, Z.-j. Liu, Y. Hu, Y. Wang, S.-j. Liu and Y. Gan, ACS Appl. Mater. Interfaces, 2016, 8, 4416-4423.

33 A. K. Singh, M. A. Hahn, L. G. Gutwein, M. C. Rule, J. A. Knapik, B. M. Moudgil, S. R. Grobmyer and S. C. Brown, Int. J. Nanomed., 2012, 7, 2739-2750.

34 G. Shen, R. Xing, N. Zhang, C. Chen, G. Ma and X. Yan, ACS Nano, 2016, 10, 5720-5729.

35 S. E. A. Gratton, P. A. Ropp, P. D. Pohlhaus, J. C. Luft, V. J. Madden, M. E. Napier and J. M. DeSimone, Proc. Natl. Acad. Sci. U. S. A., 2008, 105, 11613-11618.

36 A. Z. Wang, R. Langer and O. C. Farokhzad, Annu. Rev. Med., 2012, 63, 185-198.

37 V. Kumar, G. A. Baker and S. Pandey, ChemComm, 2011, 47, 4730-4732.

38 K. Welsher, Z. Liu, S. P. Sherlock, J. T. Robinson, Z. Chen, D. Daranciang and H. Dai, Nat. Nanotechnol., 2009, 4, 773.

39 S. Luo, E. Zhang, Y. Su, T. Cheng and C. Shi, Biomaterials, 2011, 32, 7127-7138.

40 Y. Zhang, C. Zhang, J. Chen, L. Liu, M. Hu, J. Li and H. Bi, ACS Appl. Mater. Interfaces, 2017, 9, 25152-25163.
41 Y. Liu, X. Zhang, M. Zhou, X. Nan, X. Chen and X. Zhang, ACS Appl. Mater. Interfaces, 2017, 9, 43498-43507.

42 T. L. Moore, L. Rodriguez-Lorenzo, V. Hirsch, S. Balog, D. Urban, C. Jud, B. Rothen-Rutishauser, M. Lattuada and A. Petri-Fink, Chem. Soc. Rev., 2015, 44, 6287-6305.

43 C. Zhang, T. Liu, Y. Su, S. Luo, Y. Zhu, X. Tan, S. Fan, L. Zhang, Y. Zhou, T. Cheng and C. Shi, Biomaterials, 2010, 31, 6612-6617.

44 J. Shi, P. W. Kantoff, R. Wooster and O. C. Farokhzad, Nat. Rev. Cancer, 2017, 17, 20-37.

45 M. K. Khaing Oo, Y. Yang, Y. Hu, M. Gomez, H. Du and H. Wang, ACS Nano, 2012, 6, 1939-1947.

46 A. Kumar, H. Ma, X. Zhang, K. Huang, S. Jin, J. Liu, T. Wei, W. Cao, G. Zou and X.-J. Liang, Biomaterials, 2012, 33, 1180-1189.

47 S. Behzadi, V. Serpooshan, W. Tao, M. A. Hamaly, M. Y. Alkawareek, E. C. Dreaden, D. Brown, A. M. Alkilany, O. C. Farokhzad and M. Mahmoudi, Chem. Soc. Rev., 2017, 46, 4218-4244.

48 F. Zhao, Y. Zhao, Y. Liu, X. Chang, C. Chen and Y. Zhao, Small, 2011, 7, 1322-1337.

49 J. Yang, X. Chen, Y. Li, Q. Zhuang, P. Liu and J. Gu, Chem. Mater., 2017, 29, 4580-4589.

50 M. Arduengo, Differentiating Mitochondrial Toxicity from Other Types of Mechanistic Toxicity, http:// www.promega.com/resources/pubhub/differentiatingmitochondrial-toxicity-from-other-types-of-mechanistictoxicity/, accessed Jan, 2018. 